\title{
An Improved Antenna Array Pattern Synthesis Method Using Fast Fourier Transforms
}

\author{
Xucun Wang, ${ }^{1,2}$ Yiguo Zhou, $^{1}$ and Yanfei Wang ${ }^{1}$ \\ ${ }^{1}$ Institute of Electronics, Chinese Academy of Sciences, Beijing 100190, China \\ ${ }^{2}$ University of Chinese Academy of Sciences, Beijing 100190, China \\ Correspondence should be addressed to Xucun Wang; wangxucun1900@126.com
}

Received 21 April 2014; Revised 18 August 2014; Accepted 22 August 2014

Academic Editor: Michelangelo Villano

Copyright (C) 2015 Xucun Wang et al. This is an open access article distributed under the Creative Commons Attribution License, which permits unrestricted use, distribution, and reproduction in any medium, provided the original work is properly cited.

An improved antenna array pattern synthesis method using fast Fourier transform is proposed, which can be effectively applied to the synthesis of large planar arrays with periodic structure. Theoretical and simulative analyses show that the original FFT method has a low convergence rate and the converged solution can hardly fully meet the requirements of the desired pattern. A scaling factor is introduced to the original method. By choosing a proper value for the scaling factor, the convergence rate can be greatly improved and the final solution is able to fully meet the expectations. Simulation results are given to demonstrate the effectiveness of the proposed algorithm.

\section{Introduction}

In order to solve complex antenna pattern synthesis problems, various methods using various optimization algorithms have been developed. In [1], a quadratic program is formed for arbitrary array pattern synthesis. In [2], a convex optimization problem [3] is formulated for pattern synthesis subject to arbitrary upper bounds. For certain cases, the convex programming problem can be reduced to a linear programming problem [4]. In [5] an effective hybrid optimization method is proposed for footprint pattern synthesis of very large planar antenna arrays [6]. The methods mentioned above all adopt conventional optimization algorithms [7]. Global optimization algorithms such as genetic algorithms [8-10] and particle swarm optimization algorithms [11-13] have also been successfully applied in pattern synthesis problems. To approximate the desired pattern for an array, we need to discretize the angular space. The bigger the elements number is, the greater the required discrete density needs to be. Usually, the computational complexity would grow greatly as the elements number increases. As a consequence, normal synthesis techniques using local or global optimization algorithms are usually not suitable for large planar arrays.
As we know, fast Fourier transforms (FFT) are able to quickly compute the radiation pattern of an array with periodic structure. Once the number of FFT points is specified, the computation time is barely affected by the element number. In [14], an FFT method suitable for large planar arrays is proposed. The operation is very straightforward, which mainly involves direct and inverse fast Fourier transforms. In [15], a modified iterative FFT technique is proposed for leaky-wave antenna pattern synthesis. In [16], FFT is used for the pattern synthesis of nonuniform antenna arrays. The iterative FFT method is very efficient as shown in [14], and many examples are presented, but why the method is effective has not been explained.

In this paper, both theoretical and simulative analyses of the FFT method are presented. It is found that, though effective, it is a slow-convergence method and can hardly converge to the optimum solution. Based on the analyses, we introduce a scaling factor and the performance can be greatly improved.

\section{Original FFT Method for Pattern Synthesis}

First, we establish the relationship between a certain point in the FFT result and the corresponding angle of the radiation 
pattern. Consider a planar array with $M \times N$ elements arranging in a rectangular grid and spacing $d_{x}$ and $d_{y}$ between rows and columns. Assume the element pattern is isotropic. The array factor is given by

$$
F(\theta, \phi)=\sum_{m=0}^{M-1} \sum_{n=0}^{N-1} I_{m n} e^{j 2 \pi\left[m\left(\left(d_{x} / \lambda\right) \sin \theta \cos \varphi\right)+n\left(\left(d_{y} / \lambda\right) \sin \theta \sin \varphi\right)\right]},
$$

where $I_{m n}$ is the complex excitation of the $m n$th element and $\lambda$ is the wavelength. If the $(u, v)$ coordinates $(u=$ $\sin \theta \cos \varphi, v=\sin \theta \sin \varphi$ ) are used, the array pattern can be written as

$$
F(u, v)=\sum_{m=0}^{M-1} \sum_{n=0}^{N-1} I_{m n} e^{j 2 \pi\left[m\left(\left(d_{x} / \lambda\right) u\right)+n\left(\left(d_{y} / \lambda\right) v\right)\right]} .
$$

Performing $K_{\mathrm{fft}} \times L_{\mathrm{fft}}$ points 2D inverse fast Fourier transform (IFFT) on the excitations, we get

$$
\begin{array}{r}
I F(k, l)=\frac{1}{K_{\mathrm{fft}} L_{\mathrm{fft}}} \sum_{m=0}^{M-1} \sum_{n=0}^{N-1} I_{m n} e^{j 2 \pi\left[m\left(k / K_{\mathrm{fft}}\right)+n\left(l / L_{\mathrm{ffi}}\right)\right]} \\
\left(k=0,1, \ldots, K_{\mathrm{fft}}-1 ; l=0,1, \ldots, L_{\mathrm{fft}}-1\right) .
\end{array}
$$

If we want to represent the array factor using $I F(k, l)$, then the coordinates $(u, v)$ and $(k, l)$ are related by

$$
\begin{aligned}
& \frac{d_{x}}{\lambda} u=\frac{k}{K_{\mathrm{fft}}}+S_{k} \\
& \frac{d_{y}}{\lambda} v=\frac{l}{L_{\mathrm{fft}}}+S_{l},
\end{aligned}
$$

where $S_{k}$ and $S_{l}$ are integers, making sure both sides of the equations have the same value range. Suppose that both $K_{\mathrm{fft}}$ and $L_{\mathrm{fft}}$ are even numbers. Define

$$
\begin{aligned}
& k^{\prime}= \begin{cases}k+\frac{K_{\mathrm{fft}}}{2}, & k<\frac{K_{\mathrm{fft}}}{2} \\
k-\frac{K_{\mathrm{fft}}}{2}, & k \geq \frac{K_{\mathrm{fft}}}{2}\end{cases} \\
& l^{\prime}= \begin{cases}l+\frac{L_{\mathrm{fft}}}{2}, & l<\frac{L_{\mathrm{fft}}}{2} \\
l-\frac{L_{\mathrm{fft}}}{2}, & l \geq \frac{L_{\mathrm{fft}}}{2} .\end{cases}
\end{aligned}
$$

Combining (4) and (5) and considering the value ranges of $u$ and $v$, we have

$$
\begin{aligned}
& u=\frac{\lambda}{d_{x}}\left(\frac{k^{\prime}}{K_{\mathrm{fft}}}-\frac{1}{2}\right) \\
& v=\frac{\lambda}{d_{y}}\left(\frac{l^{\prime}}{L_{\mathrm{fft}}}-\frac{1}{2}\right) .
\end{aligned}
$$

So, the relation between the array factor and the IFFT of the array excitation is

$$
F(u, v)=K_{\mathrm{fft}} L_{\mathrm{fft}} \cdot I F\left(k^{\prime}, l^{\prime}\right) .
$$

Finally, the visible space is given by

$$
\left(\frac{\lambda}{d_{x}}\right)^{2}\left(\frac{k^{\prime}}{K_{\mathrm{fft}}}-\frac{1}{2}\right)^{2}+\left(\frac{\lambda}{d_{y}}\right)^{2}\left(\frac{l^{\prime}}{L_{\mathrm{fft}}}-\frac{1}{2}\right)^{2} \leq 1 .
$$

Note that the indices change in (5) is in fact the fftshift operation in Matlab.

Once the corresponding relationship is established, the procedure of the FFT method for pattern synthesis is given as follows.

(1) Specify dimension of the array $M \times N$, the initial excitation $I_{m n}^{(1)}$, the FFT points $K_{\mathrm{fft}} \times L_{\mathrm{fft}}$, and the maximum iteration times $N_{\max }$.

(2) Perform IFFT on the excitation of the ith iteration $I_{m n}^{(i)}$ and obtain the array factor $F_{k l}^{(i)}$.

(3) Extract the amplitude $\left|F_{k l}^{(i)}\right|$ and phase $\varphi_{k l}^{(i)}$ of $F_{k l}^{(i)}$.

(4) Compare $\left|F_{k l}^{(i)}\right|$ with the desired pattern $F_{k l}^{e}$. If the computed pattern fully meets the requirements or the maximum iteration time is reached, terminate the procedure; otherwise go to the next step.

(5) Obtain the new pattern $F_{k l}^{(i) '}$ by replacing the undesired $F_{k l}^{(i)}$ with $F_{k l}^{e} \cdot e^{j \varphi_{k l}^{(i)}}$ as follows:

$$
F_{k l}^{(i)^{\prime}}= \begin{cases}F_{k l}^{e} \cdot e^{j \varphi_{k l}^{(i)}}, & (k, l) \in U \\ F_{k l}^{(i)}, & (k, l) \notin U,\end{cases}
$$

where $U$ is a set containing all the points where the pattern is undesired. For example, if $\left(k^{*}, l^{*}\right)$ is a point within the side lobe region and $\left|F_{k^{*} l^{*}}^{(i)}\right|>F_{k^{*} l^{*}}^{e}$, then it means that the side lobe level exceeds the desired level and that $\left(k^{*}, l^{*}\right) \in U$.

(6) Perform $K_{\mathrm{fft}} \times L_{\mathrm{fft}}$ points 2-D FFT on $F_{k l}^{(i)}{ }^{\prime}$ and choose the first $M \times N$ points as the initial excitation for the next iteration $I_{m n}^{(i+1)}$. Constraints can be easily made for amplitude-only or phase-only synthesis.

(7) Go to step (2).

The procedure is also illustrated in Figure 1.

\section{Algorithm Analysis}

The procedure is simple yet effective. In this section, the method is carefully examined. Without loss of generality, take an $N$-element linear array for example. Suppose that in the $i$ th iteration the excitation sequence is $\mathbf{I}^{(i)}$ which has been extended to $M_{\mathrm{fft}}\left(M_{\mathrm{fft}}>N\right)$ points by zero padding. $M_{\mathrm{fft}}$ is the number of the FFT points. After comparing the obtained pattern $\mathbf{F}^{(i)}$ with the desired pattern, we can obtain the error pattern $\Delta \mathbf{F}^{(i)}$. Now we will find the relation between the error pattern in the next iteration $\Delta \mathbf{F}^{(i+1)}$ and $\Delta \mathbf{F}^{(i)}$. It is difficult to 


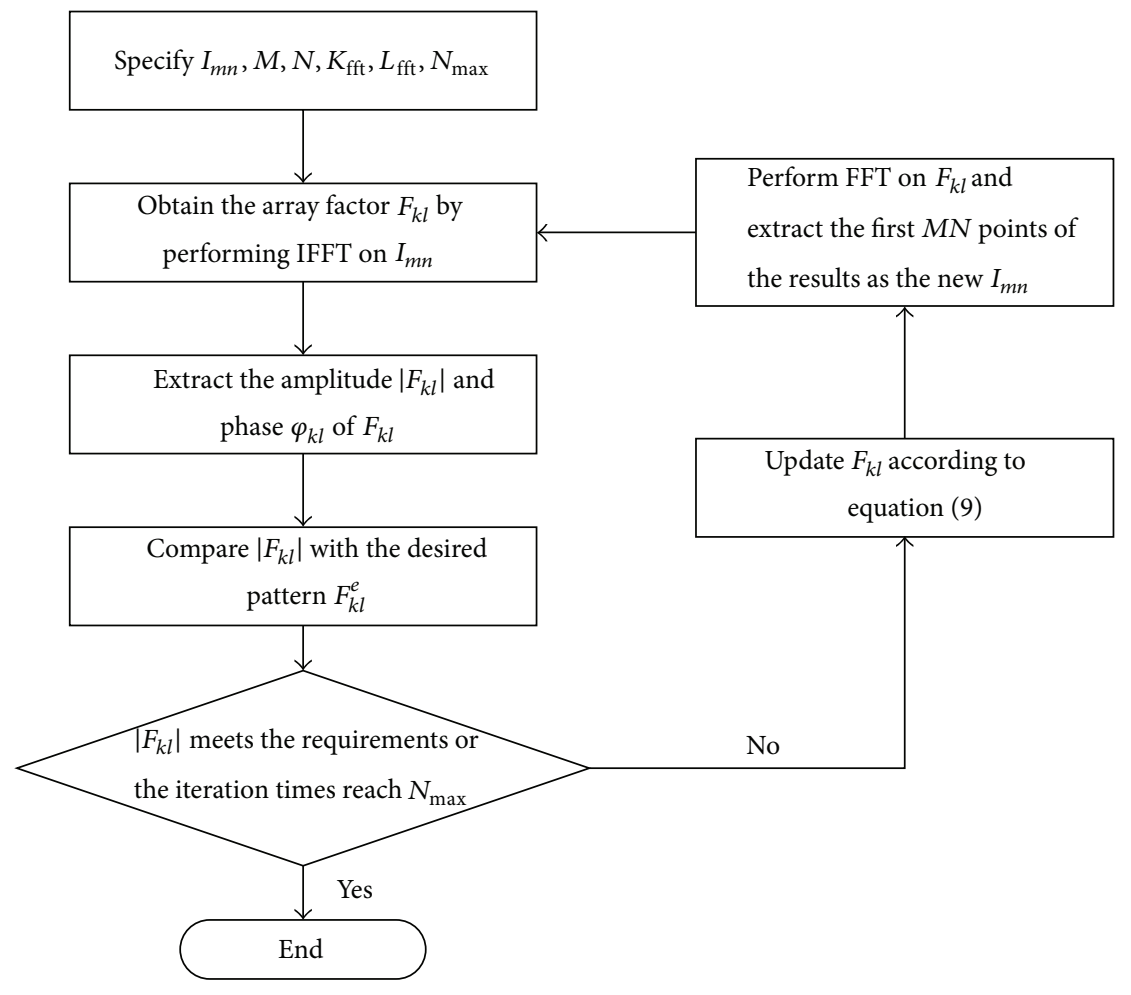

FIGURE 1: Flowchart of the FFT procedure for pattern synthesis.

precisely compute $\Delta \mathbf{F}^{(i+1)}$ through the above assumption. Let $\Delta \mathbf{I}^{(i)}$ be the corresponding error excitation for $\Delta \mathbf{F}^{(i)}$. Then

$$
\Delta \mathbf{F}^{(i)}=\operatorname{IFFT}\left[\Delta \mathbf{I}^{(i)}\right]
$$

where $\Delta \mathbf{I}^{(i)}$ contains $M_{\mathrm{fft}}$ points. Consider the elements in $\Delta \mathbf{F}^{(i)}$

$$
\Delta F_{m}^{(i)}= \begin{cases}\left(F_{m}^{e}-\left|F_{m}^{(i)}\right|\right) \cdot e^{j \varphi_{m}^{(i)}}, & m \in U \\ 0, & m \notin U .\end{cases}
$$

If the set $U$ only contains points representing the side lobe region, then the magnitude values of the elements in $\Delta \mathbf{F}^{(i)}$ are very small, as well as $\Delta \mathbf{I}^{(i)}$. So the variation in $\mathbf{I}^{(i+1)}$ from $\mathbf{I}^{(i)}$ is very little. Then we can use $\mathbf{F}^{(i)}+\Delta \mathbf{F}^{(i)}$ as the desired pattern in the $i+1$ th iteration and obtain the error pattern

$$
\Delta \mathbf{F}^{(i+1)} \approx \mathbf{F}^{(i)}+\Delta \mathbf{F}^{(i)}-\mathbf{F}^{(i+1)}=\operatorname{IFFT}\left[\mathbf{I}^{(i)}+\Delta \mathbf{I}^{(i)}-\mathbf{I}^{(i+1)}\right] .
$$

For complex weighting using both amplitude and phase, $\mathbf{I}^{(i+1)}$ is given as

$$
\mathbf{I}^{(i+1)}=\mathbf{I}^{(i)}+\left[\Delta \mathbf{I}^{(i)}-\Delta \mathbf{I}^{(i)^{\prime}}\right]
$$

where $\Delta \mathbf{I}^{(i)^{\prime}}$ is a sequence by setting the first $N$ elements in $\Delta \mathbf{I}^{(i)}$ to zero. So

$$
\Delta \mathbf{F}^{(i+1)} \approx \operatorname{IFFT}\left[\Delta \mathbf{I}^{(i)^{\prime}}\right]
$$

As a result, we have

$$
\left\|\Delta \mathbf{F}^{(i+1)}\right\|_{2}^{2} \approx \frac{1}{M_{\mathrm{fft}}}\left\|\Delta \mathbf{I}^{(i)^{\prime}}\right\|_{2}^{2} \leq \frac{1}{M_{\mathrm{fft}}}\left\|\Delta \mathbf{I}^{(i)}\right\|_{2}^{2}=\left\|\Delta \mathbf{F}^{(i)}\right\|_{2}^{2},
$$

where $\|\cdot\|_{2}$ denotes the $l_{2}$-norm.

It is seen from (15) that the method has the ability to converge. However, as $M_{\mathrm{fft}}$ increases the difference between $\left\|\Delta \mathbf{I}^{(i)^{\prime}}\right\|_{2}^{2}$ and $\left\|\Delta \mathbf{I}^{(i)}\right\|_{2}^{2}$ decreases. We can conclude that the method has a lower converge rate for larger FFT points. For amplitude-only synthesis, the analysis is similar and (15) still holds. For phase-only analysis, the excitation of the $i+1$ th iteration is

$$
\mathbf{I}^{(i+1)}=\mathbf{I} \cdot e^{j \cdot \arg \left[\mathbf{I}^{(i)}+\Delta \mathbf{I}^{(i)}-\Delta \mathbf{I}^{(i)}\right)^{\prime}},
$$

where $\mathbf{I}$ is the given amplitude. Since the magnitude value of $\Delta \mathbf{I}^{(i)}$ can be very small compared to $\mathbf{I}, \mathbf{I}^{(i+1)}$ can be approximated by

$$
\mathbf{I}^{(i+1)} \approx \mathbf{I}^{(i)}+\left[\Delta \mathbf{I}^{(i)}-\Delta \mathbf{I}^{(i)^{\prime}}\right] .
$$

So the convergence properties are similar to amplitude-only or complex tapering.

To verify our analysis, we will give an example. Consider a linear array with 60 elements spacing by half wavelength. For the amplitude-only synthesis, the desired pattern has a maximum side lobe level of $-40 \mathrm{~dB}$. For the phase-only case, the maximum side lobe level is $-18 \mathrm{~dB}$. We use the uniform taper as the initial excitation and set the maximum iteration 


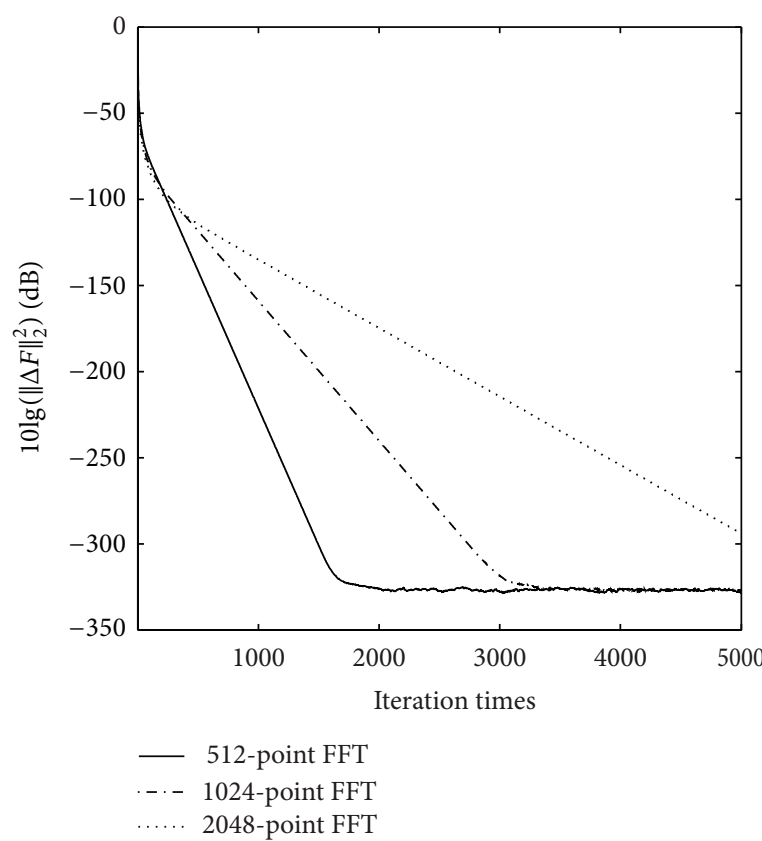

(a)

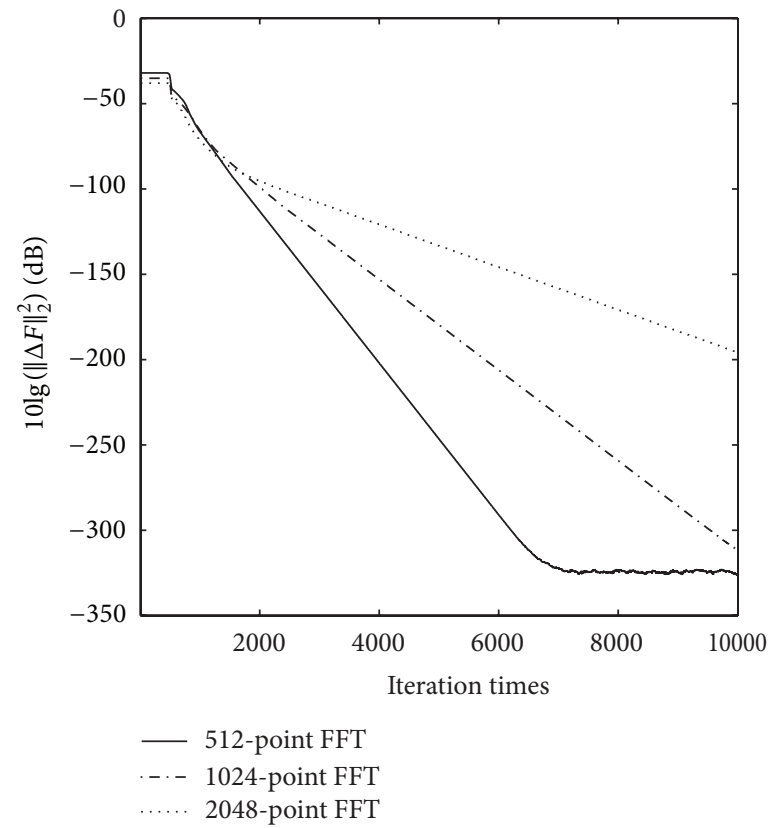

(b)

FIgURE 2: Norm of the error pattern: (a) amplitude-only and (b) phase-only.

number to 5000 and 10000 for the two cases, respectively. Figure 2 shows the convergence properties of the norm of the error pattern. We can see that the method has a low convergence rate, as it converges logarithmically in the latter part of the iteration. We can also see that the bigger the FFT points number is, the lower the method converges. Figure 2 also illustrates that although the norm of the error pattern can converge to a very low value, it does not reach zero. It means that the method can hardly synthesize a pattern that fully meets the requirements of the desired pattern.

\section{Improved FFT Method for Pattern Synthesis}

For planar arrays, the elements in the error pattern $\Delta \mathbf{F}^{(i)}$ are given by

$$
\Delta F_{k l}^{(i)}= \begin{cases}\left(F_{k l}^{e}-\left|F_{k l}^{(i)}\right|\right) \cdot e^{j \varphi_{k l}^{(i)}}, & (k, l) \in U \\ 0, & (k, l) \notin U .\end{cases}
$$

First we divide $U$ into $U_{s}$ and $U_{m}$, which are the sets representing the side lobe region and the main lobe region, respectively. When $(k, l) \in U_{s}, F_{k l}^{e}-\left|F_{k l}^{(i)}\right|<0$ and the value tends to converge towards zero more slowly as the procedure iterates. So, to change the slowly varying characteristics, we propose the following updating equation for the error pattern:

$$
\Delta F_{k l}^{(i)}= \begin{cases}\left(\alpha^{(i)} \cdot F_{k l}^{e}-\left|F_{k l}^{(i)}\right|\right) \cdot e^{j \varphi_{k l}^{(i)}}, & (k, l) \in U_{s} \\ \left(F_{k l}^{e}-\left|F_{k l}^{(i)}\right|\right) \cdot e^{j \varphi_{k l}^{(i)}}, & (k, l) \in U_{m} \\ 0, & (k, l) \notin U,\end{cases}
$$

where $\alpha^{(i)}$ is a scaling factor within the range $[0,1]$. The smaller the value of $\alpha^{(i)}$ is, the more intense the excitation changes. In (19), when $(k, l) \in U_{m}$, the error pattern remains unchanged, since for main lobe shaping synthesis, we need to approximate $\left|F_{k l}^{(i)}\right|-F_{k l}^{e}$ to a certain error level, whereas in the side lobe region, only $F_{k l}^{e} \geq\left|F_{k l}^{(i)}\right|$ is required. So, the final updating equation replacing (9) is given by

$$
F_{k l}^{(i)^{\prime}}= \begin{cases}\alpha^{(i)} \cdot F_{k l}^{e} \cdot e^{j \varphi_{k l}^{(i)}}, & (k, l) \in U_{s} \\ F_{k l}^{e} \cdot e^{j \varphi_{k l}^{(i)}}, & (k, l) \in U_{m} \\ F_{k l}^{(i)}, & (k, l) \notin U .\end{cases}
$$

To analyze the convergence properties of the proposed method, we still use the prior examples and consider a linear array with 60 elements spacing by half wavelength. The maximum iteration times are 5000 and 10000 as before. As illustrated in Figures 3 and 4, although the curves are not smooth, the norm of the error pattern can converge to zero for all the cases. In both figures, we can also see that the iteration times of the $\alpha=0$ case are much less than that of $\alpha=0.99$. Figures 5 and 6 show the influence of $\alpha$ value on the maximum iteration times and the array directivity. It is shown that as $\alpha$ grows near one, the iterations times increase rapidly but the directivity only changes a little. So we can say that the proposed method can give a good performance by setting $\alpha$ to zero. The radiation patterns obtained using $\alpha=0.0$ are shown in Figure 7, where $\theta$ is measured from one end of the linear array to the other end. 


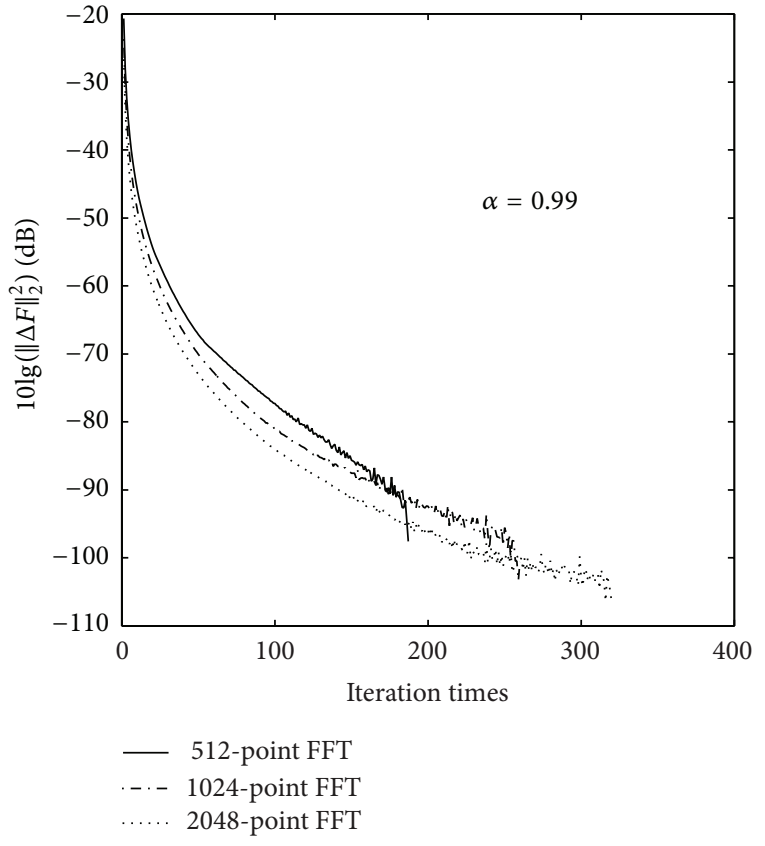

(a)

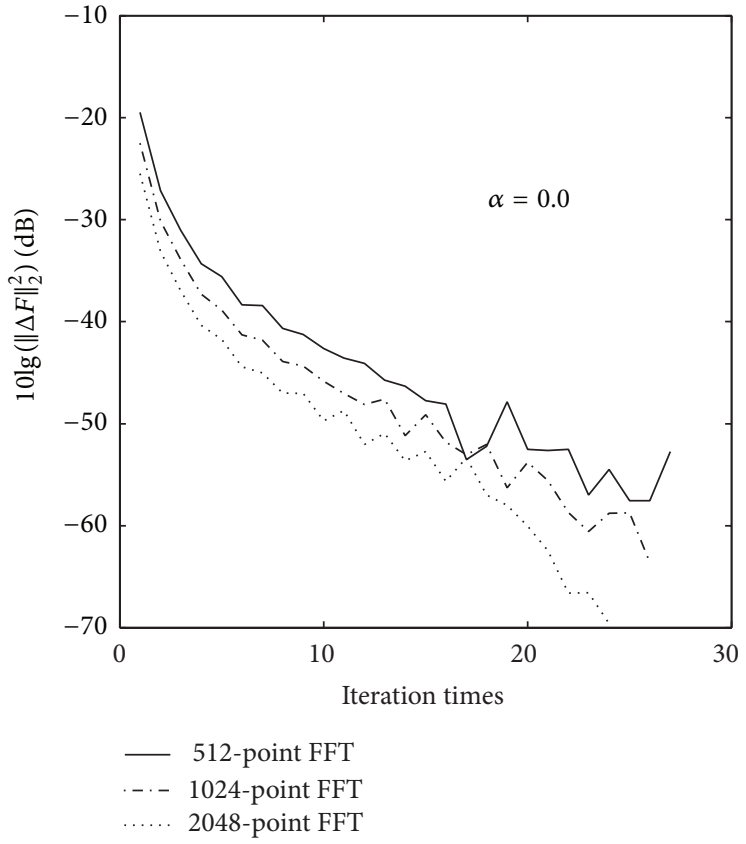

(b)

FIGURE 3: Norm of the error pattern for the amplitude-only tapering: (a) $\alpha=0.99$ and (b) $\alpha=0.0$.

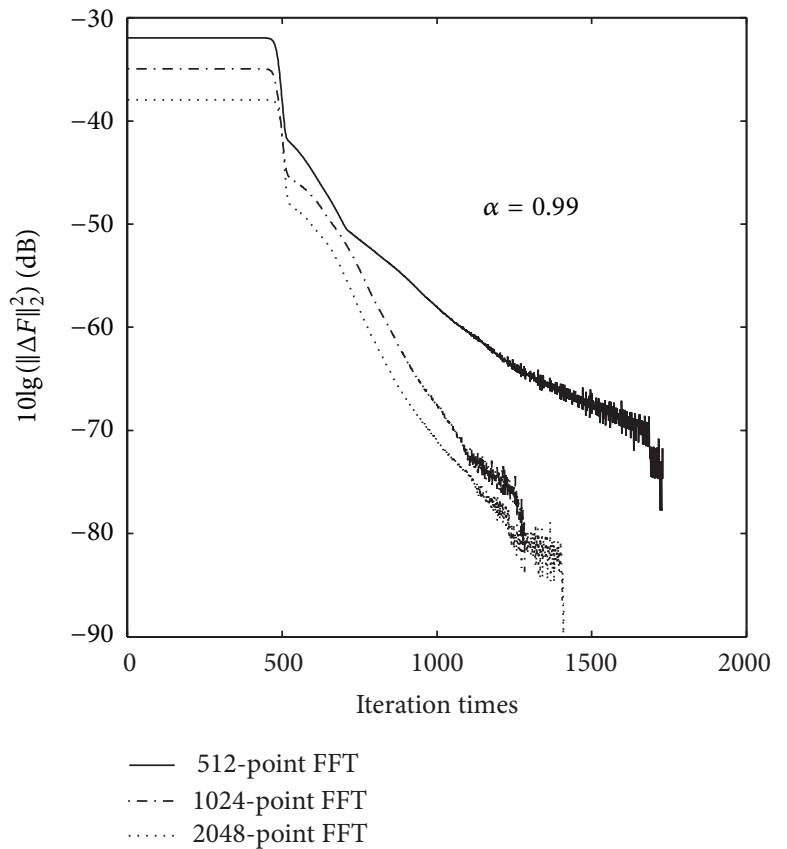

(a)

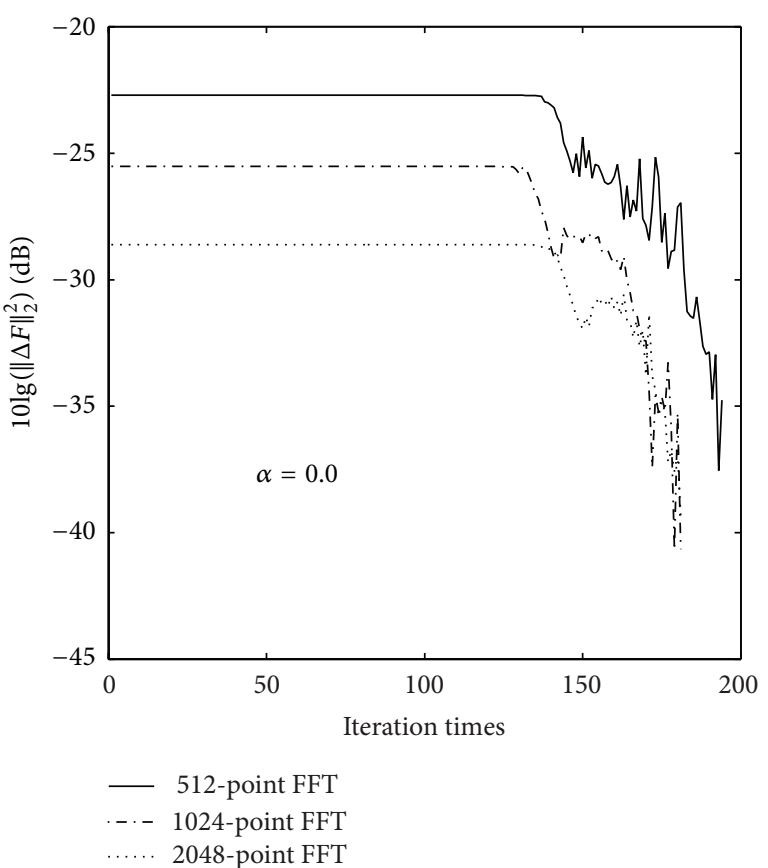

(b)

FIGURE 4: Norm of the error pattern for the phase-only tapering: (a) $\alpha=0.99$ and (b) $\alpha=0.0$.

\section{Example for Planar Array Pattern Synthesis}

Consider a $60 \times 60$ planar array with elements spacing by $0.65 \lambda$ in both directions. The desired pattern has a maximum side lobe level of $-40 \mathrm{~dB}$ and two $-60 \mathrm{~dB}$ notches at rectangular sectors $\{-0.1 \leq u \leq 0.1,0.2 \leq v<0.3\}$ and $\{0.3 \leq u \leq 0.4,-0.1 \leq v<0.1\}$. The procedure suggested in [14] is adopted. First the $256 \times 256$ points FFT is used. At the following two phases, $512 \times 512$ and $1024 \times 1024$ points FFT are performed, with the previous results being 


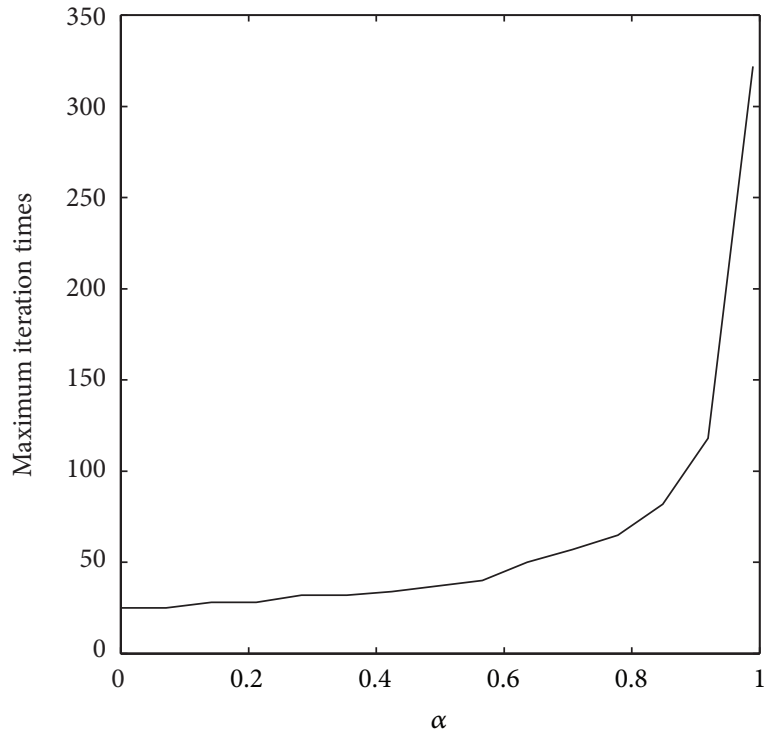

— 2048-point FFT

(a)

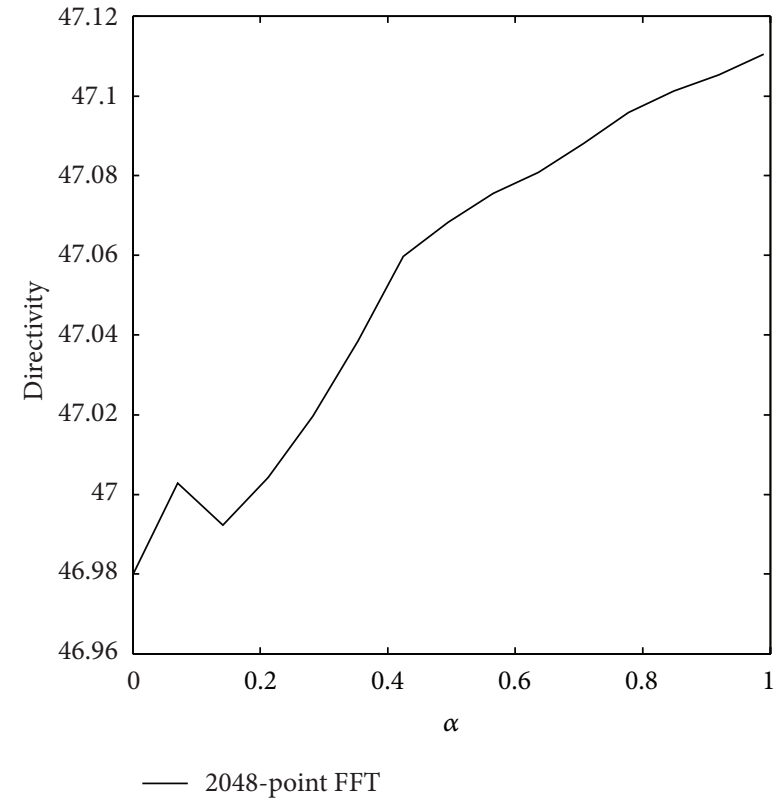

(b)

FIGURE 5: Maximum iteration times (a) and directivity of the array (b) versus $\alpha$ for the amplitude-only tapering.

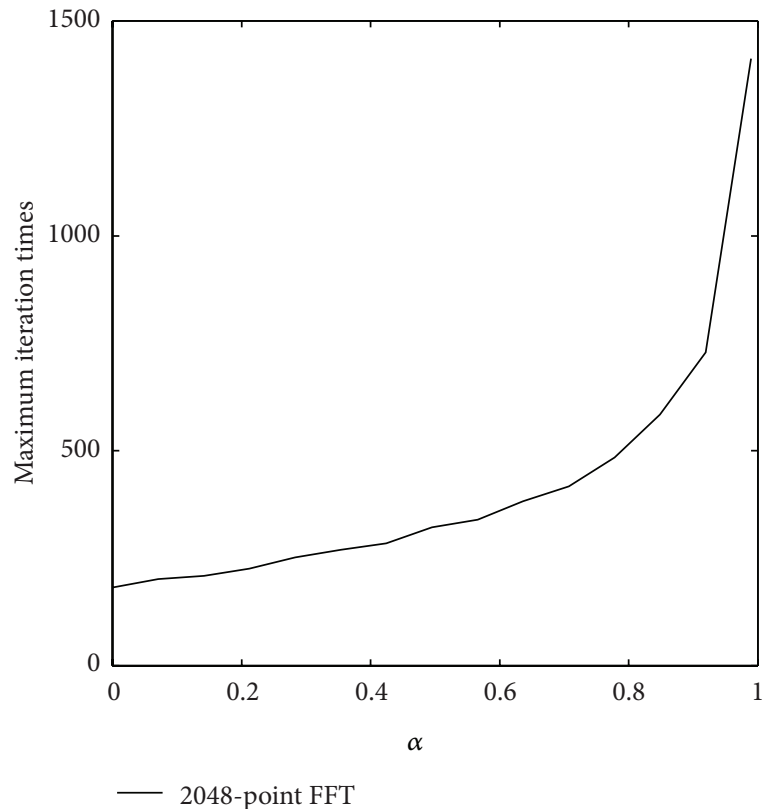

(a)

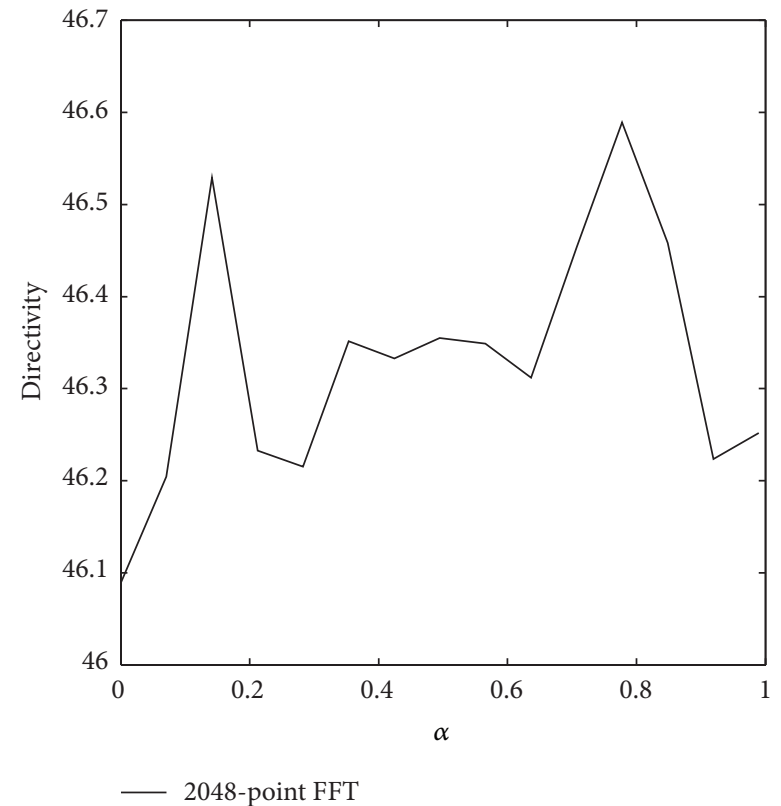

(b)

FIGURE 6: Maximum iteration times (a) and directivity of the array (b) versus $\alpha$ for the phase-only tapering.

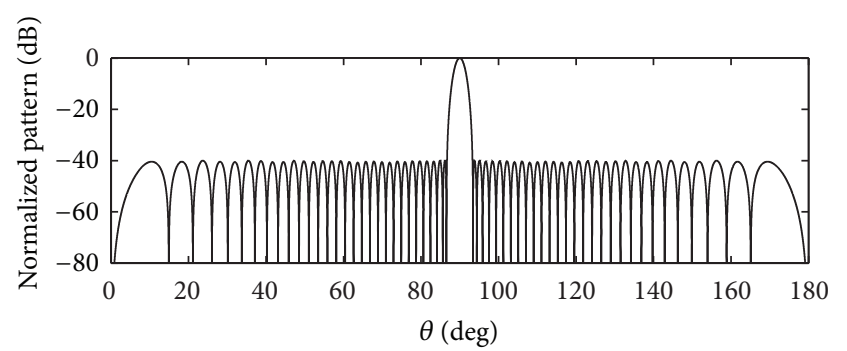

(a)

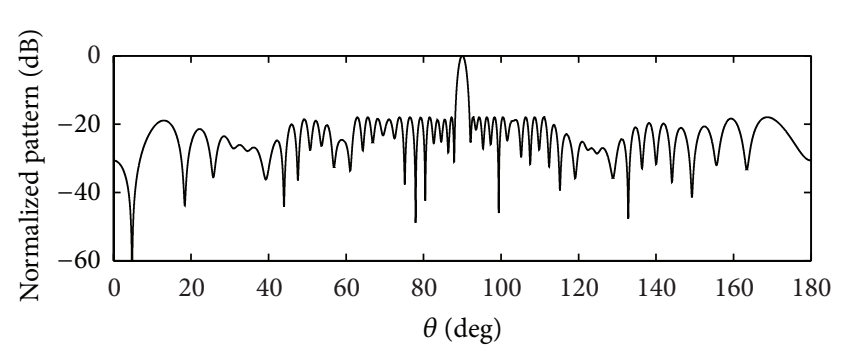

(b)

FIGURE 7: Normalized pattern: (a) amplitude-only tapering and (b) phase-only tapering. 

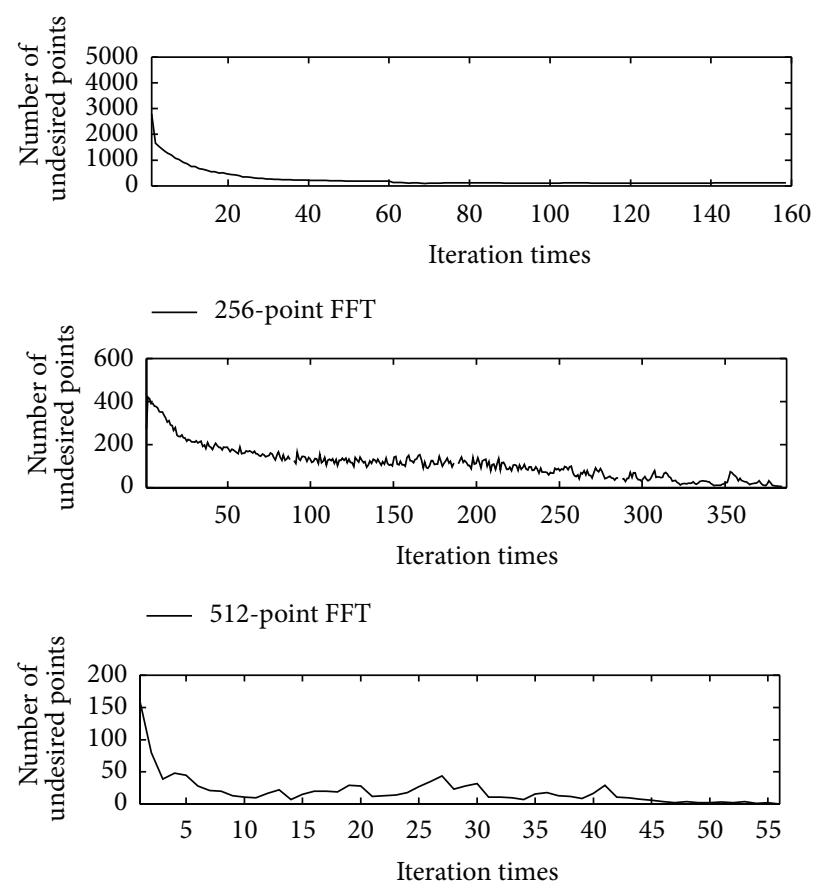

— 1024-point FFT

FIGURE 8: Number of undesired points versus iteration times.
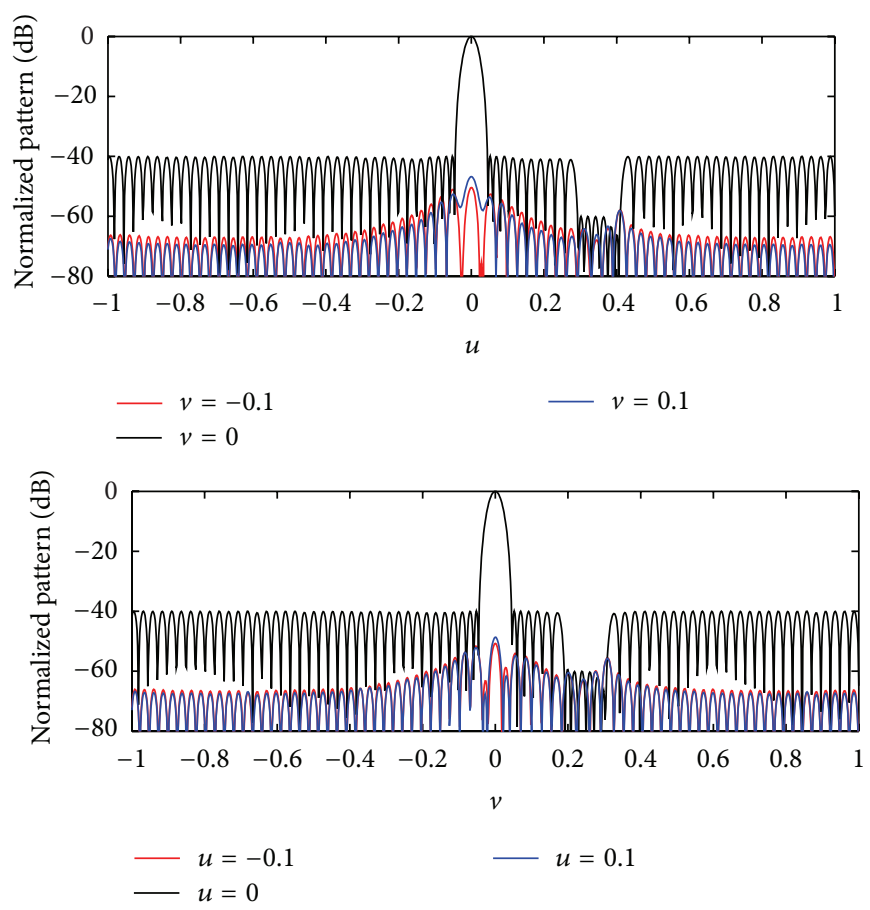

FIGURE 9: Normalized pattern at $u$-cut and $v$-cut planes.

the initial excitations for the next phase. At each phase, the maximum iteration time is 1000 , and the scaling factor $\alpha$ is set to zero. Figure 8 gives the convergence property of the number of undesired radiation points. It shows that, after the previous two phases, the final phase converges very quickly.
The $1 \mathrm{D}$ radiation patterns containing the two notch sectors are shown in Figure 9. Figure 10 presents the 2D radiation pattern, where the visible space is determined by (8). The normalized amplitude and phase of the excitations are given in Figure 11. 


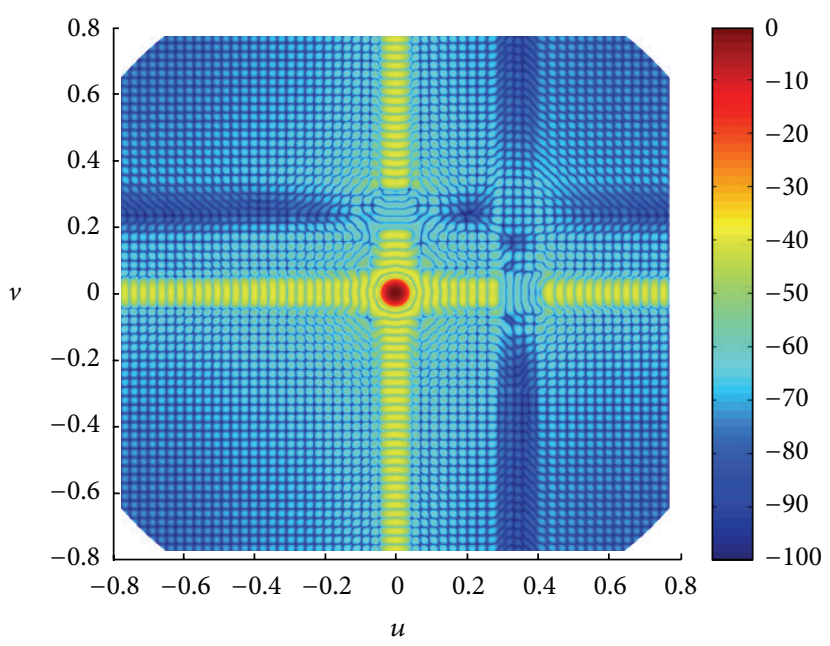

FIGURE 10: 2D radiation pattern.
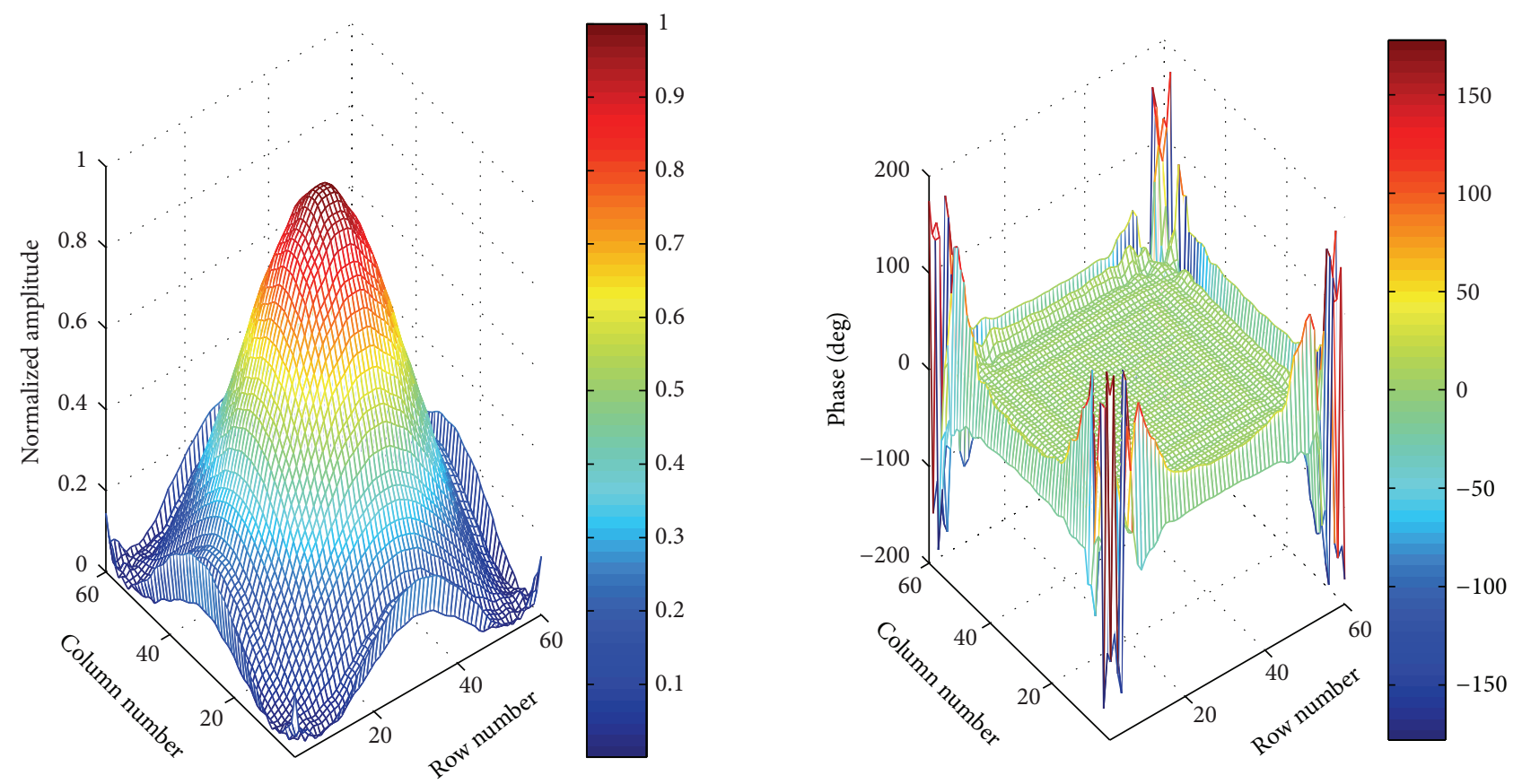

FIGURE 11: Normalized amplitude and phase of the excitations.

\section{Conclusion}

The iterative FFT method is capable of synthesizing a large planar array. In this paper, the method is validated by theoretical and simulative analyses. But the original method has a low convergence rate, and the synthesized results are usually unable to achieve the optimal solution. A scaling factor is introduced to form an improved method, which can avoid the drawbacks of the original method. Analysis and simulation results showed the effectiveness of the improved method.

\section{Conflict of Interests}

The authors declare that there is no conflict of interests regarding the publication of this paper.

\section{References}

[1] B. P. Ng, M. H. Er, and C. Kot, "Flexible array synthesis method using quadratic programming," IEEE Transactions on Antennas and Propagation, vol. 41, no. 11, pp. 1541-1550, 1993. 
[2] T. Isernia, P. D. Iorio, and F. Soldovieri, "An effective approach for the optimal focusing of array fields subject to arbitrary upper bounds," IEEE Transactions on Antennas and Propagation, vol. 48, no. 12, pp. 1837-1847, 2000.

[3] S. Boyd and L. Vandenberghe, Convex Optimization, Cambridge University Press, 2009.

[4] O. M. Bucci, L. Caccavale, and T. Isernia, "Optimal far-field focusing of uniformly spaced arrays subject to arbitrary upper bounds in nontarget directions," IEEE Transactions on Antennas and Propagation, vol. 50, no. 11, pp. 1539-1554, 2002.

[5] F. Ares, J. Fondevila-Gomez, G. Franceschetti, E. MorenoPiquero, and J. A. Rodriguez-Gonzalez, "Synthesis of very large planar arrays for prescribed footprint illumination," IEEE Transactions on Antennas and Propagation, vol. 56, no. 2, pp. 584-589, 2008.

[6] D. H. Schaubert, A. O. Boryssenko, A. van Ardenne, J. G. B. de Vaate, and C. Craeye, "The square kilometer array (SKA) antenna," in Proceedings of the IEEE International Symposium on Phased Array Systems and Technology, pp. 351-358, October 2003.

[7] J. Nocedal and S. J. Wright, Numerical Optimization, Springer, New York, NY, USA, 2006.

[8] F. J. Ares-Pena, J. A. Rodriguez-Gonzalez, E. Villanueva-Lopez, and S. R. Rengarajan, "Genetic algorithms in the design and optimization of antenna array patterns," IEEE Transactions on Antennas and Propagation, vol. 47, no. 3, pp. 506-510, 1999.

[9] D. Marcano and F. Durán, "Synthesis of antenna arrays using genetic algorithms," IEEE Antennas and Propagation Magazine, vol. 42, no. 3, pp. 12-20, 2000.

[10] L. Shi, Y. Deng, H. Sun, R. Wang, J. Ai, and H. Yan, "An improved real-coded genetic algorithm for the beam forming of spaceborne SAR," IEEE Transactions on Antennas and Propagation, vol. 60, no. 6, pp. 3034-3040, 2012.

[11] W. T. Li, X. W. Shi, and Y. Q. Hei, "An improved particle swarm optimization algorithm for pattern synthesis of phased arrays," Progress in Electromagnetics Research, vol. 82, pp. 319-332, 2008.

[12] K. V. Deligkaris, Z. D. Zaharis, D. G. Kampitaki, S. K. Goudos, I. T. Rekanos, and M. N. Spasos, "Thinned planar array design using boolean PSO with velocity mutation," IEEE Transactions on Magnetics, vol. 45, no. 3, pp. 1490-1493, 2009.

[13] T. H. Ismail and Z. M. Hamici, "Array pattern synthesis using digital phase control by quantized particle swarm optimization," IEEE Transactions on Antennas and Propagation, vol. 58, no. 6, pp. 2142-2145, 2010.

[14] W. P. M. N. Keizer, "Fast low-sidelobe synthesis for large planar array antennas utilizing successive fast fourier transforms of the array factor," IEEE Transactions on Antennas and Propagation, vol. 55, no. 3, pp. 715-722, 2007.

[15] J. L. Gomez-Tornero, A. J. Martinez-Ros, and R. VerduMonedero, "FFT synthesis of radiation patterns with wide nulls using tapered leaky-wave antennas," IEEE Antennas and Wireless Propagation Letters, vol. 9, pp. 518-521, 2010.

[16] K. Yang, Z. Zhao, and Q. H. Liu, "Fast pencil beam pattern synthesis of large unequally spaced antenna arrays," IEEE Transactions on Antennas and Propagation, vol. 61, no. 2, pp. 627-634, 2013. 

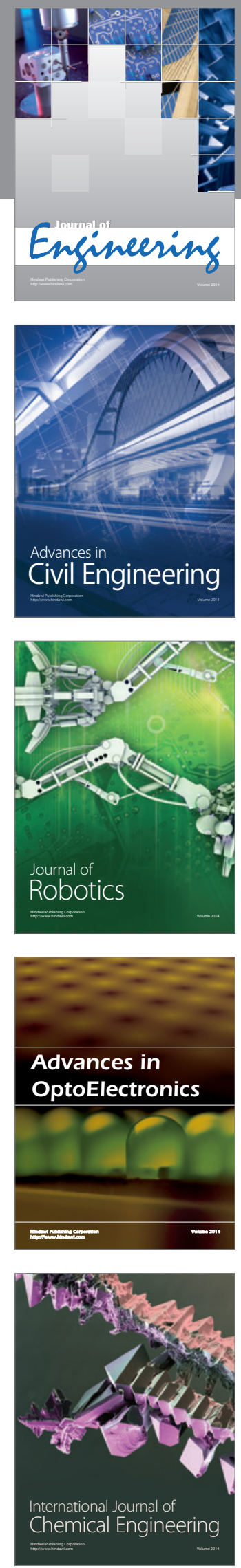

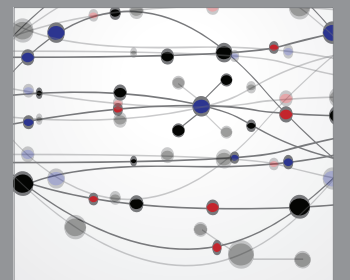

The Scientific World Journal
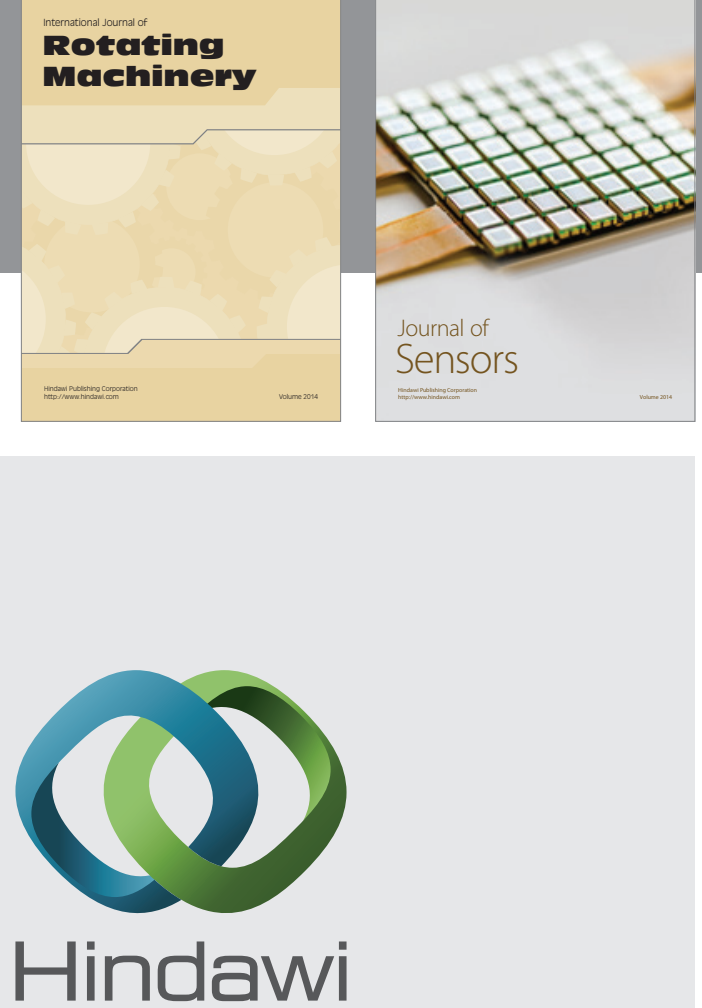

Submit your manuscripts at http://www.hindawi.com
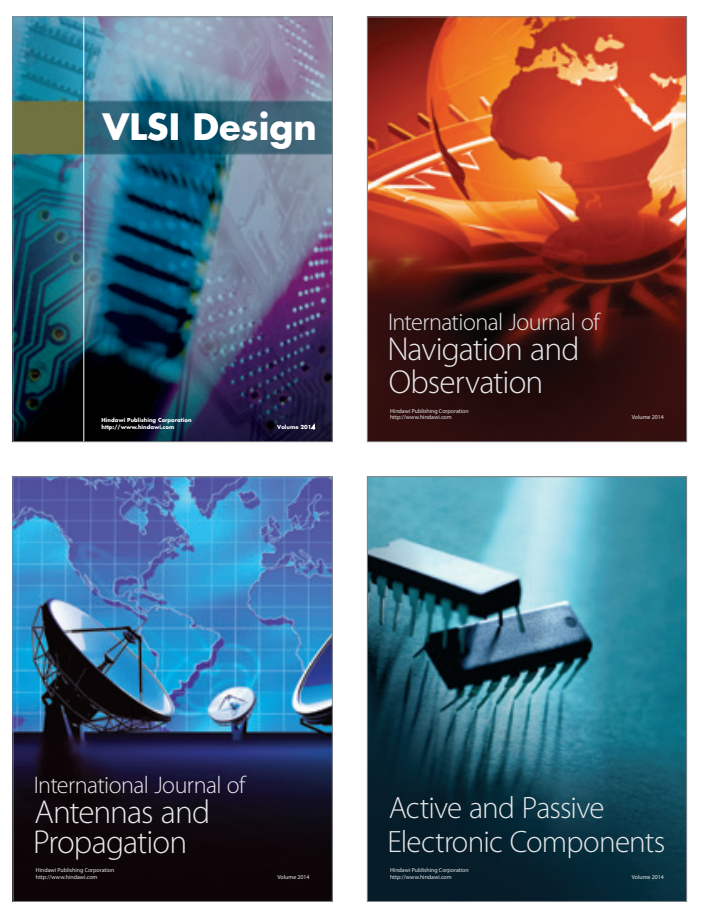
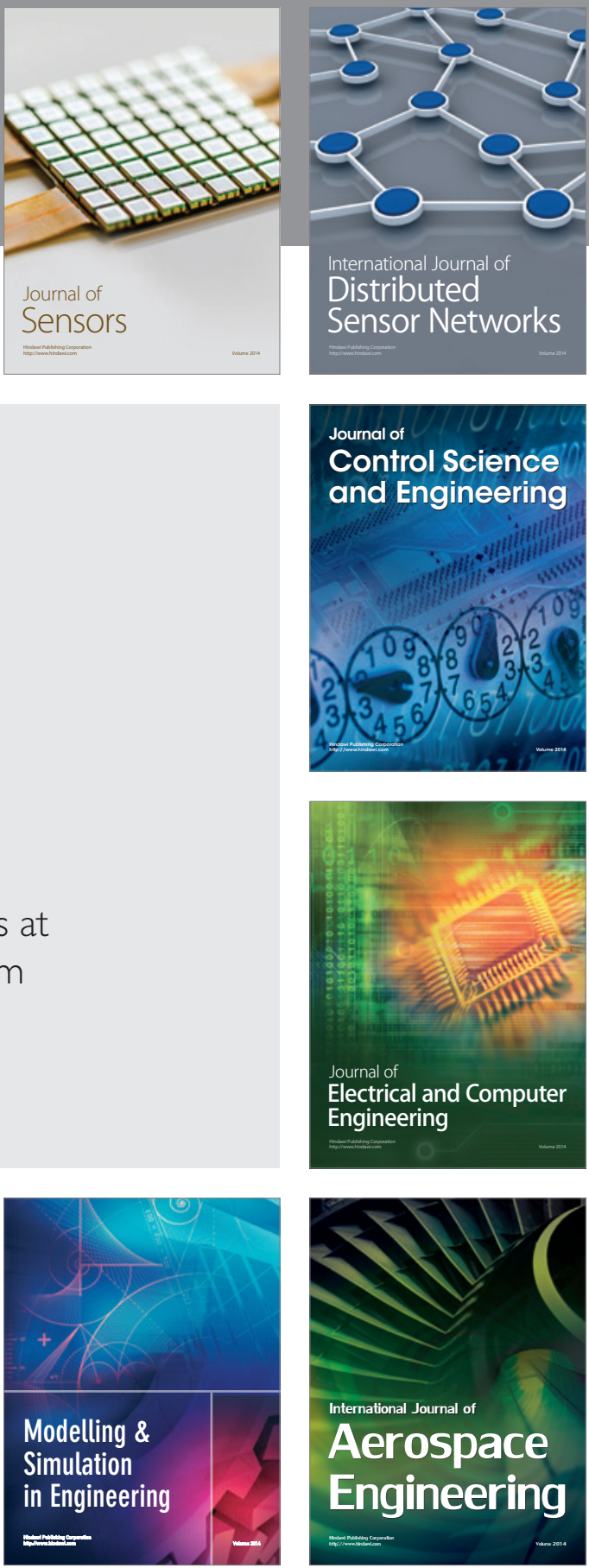

Journal of

Control Science

and Engineering
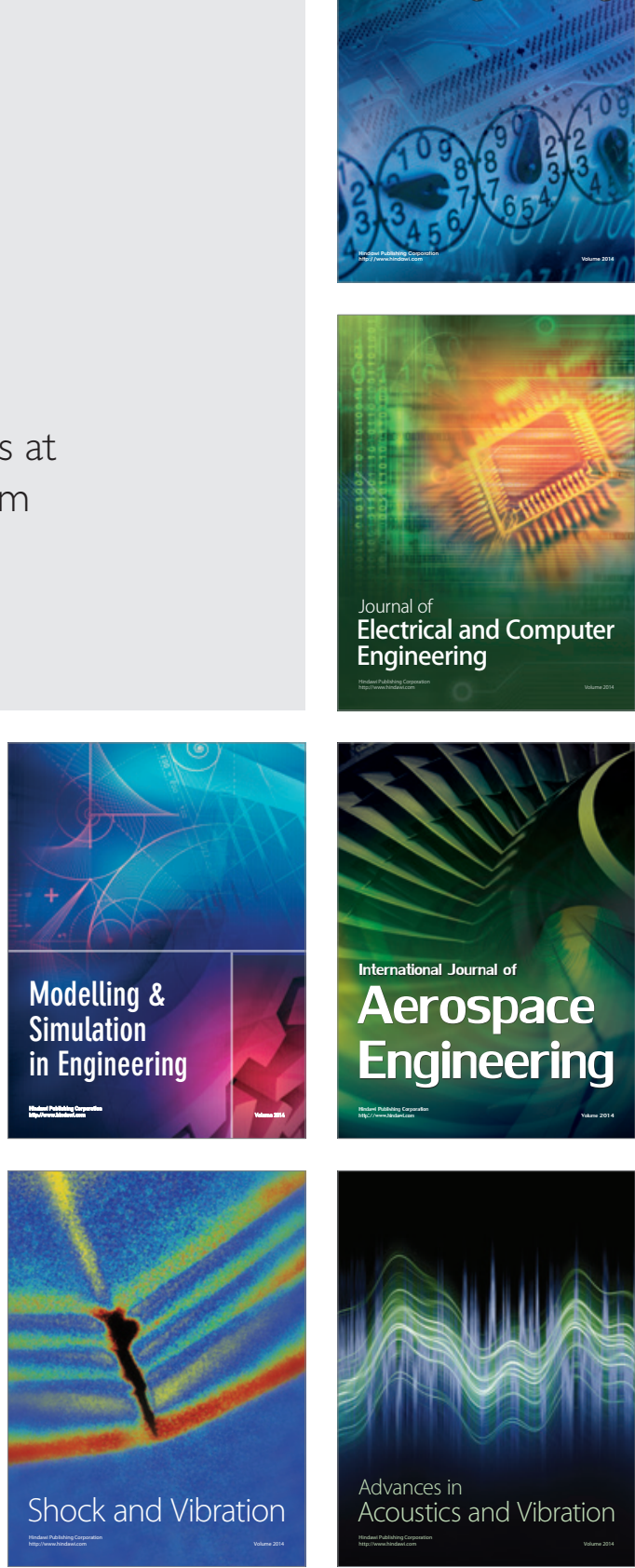